\title{
Suplementação de boro em diferentes épocas: Efeito no rendimento e na qualidade de sementes de trigo
}

\section{Boron supplementation at different times: Effect on yield and quality of wheat seeds}

\author{
André Pich Brunes ${ }^{1}$, Elisa Souza Lemes ${ }^{2}$, André Oliveira de Mendonça ${ }^{3}$, Sandro de Oliveira ${ }^{4}$, Francisco Amaral Villela ${ }^{5}$
}

Resumo: O objetivo do trabalho foi avaliar a influência da aplicação de boro em diferentes épocas no rendimento e na qualidade de sementes de trigo. O delineamento experimental utilizado foi inteiramente casualizado em esquema fatorial A X B, sendo o fator A: cultivares FUNDACEP Horizonte e BRS Campeiro, e fator B: época de aplicação, (Sem aplicação de boro, aplicação na semeadura, no perfilhamento, na floração e 15 dias após floração). A dose de boro aplicada, via solo, em todas as épocas foi de $10 \mathrm{~kg} \mathrm{ha}^{-1}$ na forma de tetraborato de sódio (boráx). Após a colheita, avaliaram-se número de espigas, número de sementes por planta, rendimento de sementes por planta, peso hectolítrico e peso de mil sementes. A qualidade fisiológica das sementes produzidas foi avaliada por testes de vigor e de germinação. A adubação de trigo com $10 \mathrm{~kg}^{-1} \mathrm{de}$ boro na forma de bórax nas diferentes épocas de aplicação, não prejudica rendimento, germinação e vigor das sementes das cultivares de trigo FUNDACEP Horizonte e BRS Campeiro. O peso volumétrico das sementes só teve redução na aplicação no perfilhamento para a cultivar FUNDACEP Horizonte e na floração para a cultivar BRS Campeiro.

Palavras-chave: Triticum aestivum L.; micronutriente; vigor; germinação.

Abstract: The aim of this study was to evaluate the effect of boron application at different times on the yield and seeds quality of wheat. The experimental design was a completely randomized factorial AXB, with factor A: FUNDACEP Horizonte and BRS Campeiro cultivars, and factor B: application time, (No application of boron, application at sowing, tillering, flowering and 15 days after flowering). The boron dose into the soil at all times was $10 \mathrm{~kg}$ ha- 1 in the form of sodium tetraborate (borax). After harvest, were evaluated number of ears, number of seeds per plant, seed yield per plant, hectoliter weight and thousand seed weight. The physiological quality of seeds produced was assessed through the vigor tests and germination test. Fertilizing wheat with $10 \mathrm{~kg}$ ha-1 of boron as borax at different times of application, without prejudice yield, germination and vigor of wheat FUNDACEP Horizonte and BRS Campeiro cultivars. The hectolitre weight of the seeds was reduced only in the application at tillering to FUNDACEP Horizonte cultivar and flowering for BRS Campeiro cultivar.

Key words: Triticum aestivum L.; micronutriente; vigor; germination.

\footnotetext{
*Autor para correspondência

Recebido para publicação em 16/02/2016; aprovado em 03/04/2016

${ }^{1}$ Eng. Agr., Doutor em Ciência \& Tecnologia de Sementes - UFPel/FAEM. beldar_brunes@msn.com

${ }^{2}$ Eng. Agr., Doutoranda do Programa de Pós-graduação Ciência \& Tecnologia de Sementes, UFPel/FAEM. Rua João Jacob Bainy, nº401A, bloco 7, ap.426,

CEP: 96065-340, Pelotas, RS, Brasil.* Autor correspondente: lemes.elisa@yahoo.com.br

${ }^{3}$ Biólogo., Doutorando do Programa de Pós-graduação Ciência \& Tecnologia de Sementes, UFPel/FAEM, andreh_mendonca@hotmail.com

${ }^{4}$ Eng. Agr., Doutorando do Programa de Pós-graduação Ciência \& Tecnologia de Sementes, UFPel/FAEM, sandrofaem@yahoo.com.br

${ }^{5}$ Prof. Dr. em Ciência \& Tecnologia de Sementes, UFPel/FAEM. francisco.villela@ ufpel.edu.br
} 


\section{INTRODUÇÃO}

O trigo (Triticum aestivum L.) é o segundo cereal mais produzido no mundo, perdendo apenas para o milho (Zea mays L.) (USDA, 2013). É uma das principais fontes de alimentação animal e humana, sendo utilizado como matériaprima na fabricação de diversos alimentos, como por exemplo, pão, bolos, biscoitos e massas, entre outros (SCHEUER et al., 2011). A estimativa de produção no Brasil para a safra 2015 é de 6,8 milhões de toneladas numa área de 2,5 milhões de hectares, dos quais o estado do Paraná e Rio Grande do Sul são responsáveis por $91 \%$ da produção (CONAB, 2015).

Dentre os fatores que podem afetar negativamente a produtividade das culturas, destaca-se a deficiência nutricional de micronutrientes. $\mathrm{O}$ suprimento balanceado de nutrientes além de favorecer o crescimento das plantas é também relevante para o seu sistema defensivo (MARSCHNER, 1996). O boro é essencial em diversas etapas do desenvolvimento da planta, sendo elemento de baixa mobilidade dentro do floema, e consequentemente, os sintomas de sua deficiência manifestam-se nos tecidos jovens ou recém-formados (MALAVOLTA et al., 1997). Este nutriente é exigido em pequenas quantidades pelas culturas, sendo a decisão para aplicar doses adequadas deste elemento no solo, vital para aumentar a produtividade, exigindo-se cautela, pois o intervalo de deficiência e toxicidez é bastante estreito (SCIVITTARO; MACHADO, 2004). Em gramíneas, o sintoma mais comum da toxidez de boro é a clorose nas margens e nas pontas de folhas (YAU; RYAN, 2008).

De acordo com Miwa e Fujiwara (2010) as exigências de $\mathrm{B}$ nas culturas podem ser distintas nas fases vegetativas e reprodutivas. Apesar do papel fisiológico desse nutriente ainda não estar perfeitamente entendido, sabe-se da sua importância na formação da parede celular, mais especificamente na síntese de componentes, como a pectina, a celulose e a lignina e no transporte de glucídios (MORAES et al., 2002) sendo sua desordem nutricional prejudicial ao crescimento radicular (OBATA, 1995). Além disso, o boro é importante na germinação do grão de pólen e crescimento do tubo polínico (KAPPES et al., 2008). Desse modo, a deficiência leva a uma má formação dos grãos dos cereais e a um baixo pegamento das flores, assim sua presença aumenta a granação e proporciona menores valores de esterilidade masculina e chochamento dos grãos.

Alguns trabalhos de pesquisa têm mostrado respostas contraditórias quanto a influência do boro sobre o rendimento e a qualidade fisiológica das sementes, como Oliveira et al. (2010), verificaram que o boro influenciou negativamente na germinação e no vigor de sementes de mamona, e que elevou a porcentagem de sementes duras e dormentes; Kappes et al. (2008) não verificaram influência da aplicação de boro na produtividade de sementes de soja. Por outro lado, Leite et al. (2011) não verificaram influência da aplicação foliar de boro na qualidade de sementes de arroz; Almeida et al. (2015) obtiveram aumento na produtividade de sementes puras de capim-mombaça com aplicação de doses de boro.

Nesse contexto, o presente trabalho teve por objetivo avaliar o efeito da aplicação de boro em diferentes épocas no rendimento e na qualidade fisiológica de sementes de trigo.

\section{MATERIAL E MÉTODOS}

O experimento foi conduzido em casa de vegetação e no Laboratório Didático de Análise de Sementes da Faculdade de Agronomia Eliseu Maciel, na Universidade Federal de Pelotas. Foram utilizadas sementes de trigo, cultivares FUNDACEP Horizonte e BRS Campeiro.

A semeadura foi realizada em vasos com capacidade de 8 litros preenchidos com solo peneirado coletado de um horizonte $\mathrm{A}_{1}$ de um PLANOSSOLO HÁPLICO Eutrófico solódico pertencente à unidade de mapeamento Pelotas (STRECK et al., 2008), o qual apresentava teor inicial de 7,25 $\mathrm{mg}$ de boro por $\mathrm{kg}$. Após o desbaste, foram deixadas 4 plantas por unidade experimental, as quais permaneceram até a colheita.

O delineamento experimental utilizado foi inteiramente casualizado, com quatro repetições. Os tratamentos constaram de um esquema fatorial A X B, sendo o fator A: cultivares FUNDACEP Horizonte e BRS Campeiro, e fator B: época de aplicação (sem a aplicação de boro, semeadura, perfilhamento, floração e 15 dias após floração). A dose de boro aplicada, via solo, em todas as épocas foi de $10 \mathrm{~kg} \mathrm{ha}^{-1}$, na forma de tetraborato de sódio (boráx), que apresenta $11,5 \%$ de boro.

A colheita foi realizada no estádio em que dois terços das espigas apresentavam coloração marrom. Após avaliaram-se as seguintes variáveis: Número de espigas (NE) e Número de sementes por planta (NSP): realizado por contagem manual das espigas e sementes em cada unidade experimental. Rendimento de sementes por planta (RSP): foi obtido pela pesagem das sementes colhidas, sendo a umidade corrigida para $13 \%$. Peso hectolítrico $(\mathrm{PH})$ : realizada com quatro repetições e balança específica, com capacidade de 1 litro de sementes, sendo o resultado expresso em $\mathrm{kg} \mathrm{hL}^{-1}$. Peso de mil sementes (PMS) - foram empregadas oito repetições de 100 sementes. Para estas pesagens calculou-se a média, o desvio padrão e o coeficiente de variação. Todas as parcelas apresentaram coeficiente de variação inferior a quatro, portanto, multiplicou-se a média por 10, e assim obteve-se o peso de mil sementes (BRASIL, 2009).

A qualidade fisiológica das sementes foi avaliada pelos seguintes testes:

Germinação $(\mathrm{G})$ : realizada com quatro repetições de 50 sementes para cada unidade experimental. A semeadura foi em substrato de papel, previamente umedecido em água destilada na proporção de 2,5 vezes o peso do papel seco e mantido em germinador à temperatura de $20{ }^{\circ} \mathrm{C}$. As avaliações foram efetuadas aos oito dias após a semeadura, conforme as Regras para Análise de Sementes (BRASIL, 2009) e os resultados expressos em porcentagem de plântulas normais.

Primeira contagem da germinação (PCG): Avaliada aos quatro dias após a semeadura por ocasião da realização do teste de germinação.

Envelhecimento acelerado (EA): realizado em caixa tipo gerbox com tela metálica. Adicionaram-se $40 \mathrm{~mL}$ de água destilada ao fundo de cada caixa, e sobre a tela foram distribuídas uniformemente as sementes de cada unidade experimental em camada única. Em seguida, as caixas, contendo as sementes, foram tampadas e acondicionadas em incubadora do tipo BOD, a $41^{\circ} \mathrm{C}$, onde permaneceram por 72 
horas. Após este período, as sementes foram submetidas ao teste de germinação (DELOUCHE; BASKIN, 1973).

Teste de frio (TF): conduzido com quatro subamostras de 50 sementes para cada unidade experimental, sendo os rolos de papel colocados em sacos plásticos, os quais foram vedados e mantidos em câmara regulada à temperatura de $10^{\circ} \mathrm{C}$ durante sete dias. Após esse período, foram transferidas para um germinador e mantidas nas mesmas condições do teste de germinação (CÍCERO; VIEIRA, 1994).

Comprimento de parte aérea (CPA) e raiz (CR): a avaliação do comprimento de parte aérea e da raiz foi realizada com quatro subamostras de 20 sementes para cada unidade experimental. Utilizou-se substrato rolo de papel para germinação do tipo "germitest", no qual as sementes foram distribuídas em duas linhas longitudinais e desencontradas no terço superior do papel. Após a confecção dos rolos, os mesmos foram colocados em germinador regulado à temperatura de $20^{\circ} \mathrm{C}$ (NAKAGAWA, 1999). No quarto dia após a semeadura, foi avaliado o comprimento da parte aérea e da raiz de dez plântulas normais escolhidas aleatoriamente, sendo cada plântula medida separadamente a parte aérea e a raiz, em seguida, foram calculadas o comprimento médio da parte aérea e da raiz.

Os dados foram analisados quanto à normalidade $\mathrm{e}$ homocedasticidade e, posteriormente, submetidos à análise de variância $(p<0,05)$. Sendo significativa a probabilidade "F", as médias de cultivar e época de aplicação foram comparadas através do teste de Tukey ambos a 5\% de probabilidade.

\section{RESULTADOS E DISCUSSÃO}

Não foi constatado efeito das diferentes épocas de aplicação de boro no número de espigas, número de sementes e peso de sementes por planta, no entanto, houve diferença entre as cultivares, sendo a BRS Campeiro superior a FUNDACEP Horizonte para essas variáveis (Tabela 1). Corroborando com os resultados obtidos por Wruck et al. (2004), onde aplicações foliares de B na pré-florada e no enchimento de sementes, não influenciaram de forma significativa no aumento da produtividade do feijoeiro. Diferindo do resultado encontrado por Lordkaew et al. (2013), que verificaram que a aplicação de boro influenciou o vingamento de grãos e o peso de grãos planta ${ }^{-1}$ em arroz.

Tabela 1. Número de espigas por planta (NEP), número de sementes por planta (NSP) e peso de sementes por planta (PSP) das cultivares de trigo FUNDACEP Horizonte e BRS Campeiro, submetidas a diferentes épocas de aplicação com boro.

\begin{tabular}{|c|c|c|c|c|c|c|c|c|c|}
\hline \multirow{2}{*}{ Época } & \multicolumn{2}{|c|}{ NEP } & \multirow{2}{*}{ Média } & \multicolumn{2}{|c|}{ NSP } & \multirow{2}{*}{ Média } & \multicolumn{2}{|c|}{ PSP (g) } & \multirow{2}{*}{ Média } \\
\hline & Horizonte & Campeiro & & Horizonte & Campeiro & & Horizonte & Campeiro & \\
\hline Sem Boro & 8,25 & 11,00 & $9,62 \mathrm{~A}$ & 316,5 & 511,9 & $414,2 \mathrm{~A}$ & 8,02 & 12,78 & $10,40 \mathrm{~A}$ \\
\hline Semeadura & 10,25 & 11,00 & $10,62 \mathrm{~A}$ & 454,7 & 405,6 & $430,1 \mathrm{~A}$ & 12,36 & 12,43 & $12,40 \mathrm{~A}$ \\
\hline Perfilhamento & 8,38 & 10,75 & $9,56 \mathrm{~A}$ & 259,7 & 496,3 & $378,0 \mathrm{~A}$ & 7,86 & 13,76 & $10,81 \mathrm{~A}$ \\
\hline Floração & 7,38 & 10,00 & $8,69 \mathrm{~A}$ & 279,4 & 410,8 & $345,1 \mathrm{~A}$ & 9,41 & 10,37 & $9,89 \mathrm{~A}$ \\
\hline $15 \mathrm{DAF}$ & 8,38 & 9,88 & $9,12 \mathrm{~A}$ & 323,6 & 418,4 & $371,0 \mathrm{~A}$ & 9,70 & 10,75 & $10,23 \mathrm{~A}$ \\
\hline Média & $8,37 \mathrm{~b}^{*}$ & $10,75 \mathrm{a}$ & & $316,5 \mathrm{~b}$ & $418,4 \mathrm{a}$ & & $9,41 \mathrm{~b}$ & $12,43 \mathrm{a}$ & \\
\hline C.V. $(\%)$ & \multicolumn{2}{|c|}{15,84} & & \multicolumn{2}{|c|}{28,36} & \multicolumn{4}{|c|}{19,36} \\
\hline
\end{tabular}

*Médias seguidas da mesma letra, minúscula na linha não diferem entre si pelo teste de $\mathrm{T}$ ( $\mathrm{p} \leq 0,05$ ), e maiúscula na coluna, não diferem entre si pelo teste Tukey $(\mathrm{p} \leq 0,05)$.

Para o peso hectolítrico (PH), a cultivar BRS Campeiro foi superior à FUNDACEP Horizonte, em todas as épocas de aplicação de boro (Tabela 2). A cultivar FUNDACEP Horizonte apresentou redução na aplicação de boro na fase de perfilhamento e 15 dias após a floração. Já a cultivar BRS Campeiro foi prejudicada na aplicação do elemento nas fases de semeadura e floração.

Tabela 2. Peso hectolítrico (PH) e peso de 1000 sementes (PMS) das cultivares de trigo FUNDACEP Horizonte e BRS Campeiro, submetidas a diferentes épocas de aplicação com boro.

\begin{tabular}{|c|c|c|c|c|c|c|}
\hline \multirow{2}{*}{ Época } & \multicolumn{2}{|c|}{$\mathrm{PH}\left(\mathrm{g} \mathrm{L}^{-1}\right)$} & \multirow{2}{*}{ Média } & \multicolumn{2}{|c|}{ P $1000(\mathrm{~g})$} & \multirow{2}{*}{ Média } \\
\hline & Horizonte & Campeiro & & Horizonte & Campeiro & \\
\hline Sem Boro & $75,97 \mathrm{~b} \mathrm{AB}$ & 79,37 a $\mathrm{A}$ & 77,67 & 46,17 a $\mathrm{A}$ & $38,76 \mathrm{~b} \mathrm{AB}$ & 42,47 \\
\hline Semeadura & $76,60 \mathrm{~b} \mathrm{~A}$ & 78,46 a BC & 77,53 & 44,03 a B & $38,80 \mathrm{~b} \mathrm{~A}$ & 41,42 \\
\hline Perfilhamento & $74,85 \mathrm{~b} \mathrm{C}$ & $78,51 \mathrm{a} A B C$ & 76,68 & 43,19 a $B$ & 38,12 b AB & 40,65 \\
\hline Floração & $76,65 \mathrm{~b} \mathrm{~A}$ & 77,85 a C & 77,25 & 45,78 a $\mathrm{A}$ & 37,07 b B & 41,42 \\
\hline $15 \mathrm{DAF}$ & $75,31 \mathrm{~b} \mathrm{BC}$ & 79,20 a $\mathrm{AB}$ & 77,25 & $44,51 \mathrm{a} A B$ & $37,64 \mathrm{~b} \mathrm{AB}$ & 41,07 \\
\hline Média & $75,97^{*}$ & 78,51 & & 44,51 & 38,12 & \\
\hline C.V. (\%) & \multicolumn{2}{|c|}{0,72} & \multicolumn{4}{|c|}{1,79} \\
\hline
\end{tabular}

*Médias seguidas da mesma letra, minúscula na linha não diferem entre si pelo teste de $\mathrm{T}$ ( $\mathrm{p} \leq 0,05$ ), e maiúscula na coluna, não diferem entre si pelo teste Tukey $(\mathrm{p} \leq 0,05)$.

O peso de mil sementes da cultivar FUNDACEP Horizonte foi superior à cultivar BRS Campeiro (Tabela 2). Este efeito pode ser atribuído à menor produtividade da cultivar FUNDACEP Horizonte em relação à cultivar BRS Campeiro, sendo as sementes maiores e mais pesadas devido a menor quantidade de drenos na planta, e consequentemente menor partição das reservas e maior acúmulo de massa por parte das sementes produzidas. Além do que a massa de mil sementes é uma característica determinada geneticamente, o que poderia justificar essa diferença entre as cultivares. A adubação com boro afetou negativamente o peso de 1000 da cultivar FUNDACEP Horizonte na aplicação quando aplicado 
nas fases de semeadura e perfilhamento. Já para a cultivar BRS Campeiro, esta foi prejudicial se efetuada na fase de floração. O nível tóxico de boro no solo e nas plantas está próximo aos níveis de deficiência, logo essa quantidade aplicada pode ter sido superior à exigência das plantas nessas fases do ciclo. Em trabalho realizado com capim-mombaça, Almeida et al. (2015) verificaram que a aplicação foliar de boro aumentou a massa de 1000 sementes. Estudando doses e épocas de aplicação foliar de boro nas características agronômicas e na qualidade de sementes de soja, Kappes et al., (2008) não observaram resultados significativos em relação à massa de 100 sementes.

No que tange à primeira contagem da germinação, a cultivar FUNDACEP Horizonte gerou sementes de maior vigor em relação à não aplicação de boro em comparação à aplicação na semeadura. No entanto, a cultivar BRS Campeiro, para a mesma variável, não foi influenciada pela aplicação (Tabela 3). Lima et al. (2013) observaram acréscimo na porcentagem de plântulas normais através do teste de primeira contagem de germinação de sementes de feijão com a aplicação de doses de boro.

Tabela 3. Primeira contagem da germinação (PCG) e germinação (G) de sementes de trigo das cultivares FUNDACEP Horizonte e BRS Campeiro, provenientes da aplicação de boro em diferentes épocas.

\begin{tabular}{|c|c|c|c|c|c|c|}
\hline \multirow{3}{*}{ Época } & \multicolumn{6}{|c|}{ Cultivar } \\
\hline & Horizonte & Campeiro & & Horizonte & Campeiro & \\
\hline & \multicolumn{2}{|c|}{ PCG $(\%)$} & Média & \multicolumn{2}{|c|}{$\mathrm{G}(\%)$} & Média \\
\hline Sem Boro & $78 \mathrm{~b} \mathrm{~B}^{*}$ & 98 a A & 88 & 99 & 100 & $100 \mathrm{~A}$ \\
\hline Semeadura & 88 a A & 95 a A & 92 & 100 & 100 & $100 \mathrm{~A}$ \\
\hline Perfilhamento & $80 \mathrm{~b} \mathrm{AB}$ & 91 a $\mathrm{A}$ & 86 & 99 & 98 & $98 \mathrm{~A}$ \\
\hline Floração & 74 b B & 95 a $\mathrm{A}$ & 85 & 99 & 99 & $99 \mathrm{~A}$ \\
\hline $15 \mathrm{DAF}$ & $82 \mathrm{a} \mathrm{AB}$ & 97 a A & 90 & 100 & 99 & $100 \mathrm{~A}$ \\
\hline Média & 80 & 95 & & $99 \mathrm{a}$ & $99 \mathrm{a}$ & \\
\hline C.V. $(\%)$ & \multicolumn{2}{|c|}{5,78} & \multicolumn{4}{|c|}{1,78} \\
\hline
\end{tabular}

*Médias seguidas da mesma letra, minúscula na linha não diferem entre si pelo teste de $\mathrm{T}(\mathrm{p} \leq 0,05)$, e maiúscula na coluna, não diferem entre si pelo teste Tukey $(\mathrm{p} \leq 0,05)$.

Já a aplicação de boro na dose de $10 \mathrm{~kg} \mathrm{ha}^{-1}$ nos diferentes estádios de desenvolvimento da cultura não interferiu na germinação das sementes produzidas na geração F1 para ambas cultivares (Tabela 3 ). Os resultados foram coerentes com Bevilaqua et al. (2002), que avaliando a adição de cálcio e boro na época de floração e pós-floração de soja, verificaram que não houve melhora na qualidade fisiológica das sementes produzidas. Resultados semelhantes foram encontrados por Ambrosano et al. (1999) que, avaliando o efeito da adubação com micronutrientes na qualidade de sementes do feijoeiro, observaram que os tratamentos não influenciaram a porcentagem de germinação e de plântulas normais, não tendo estes, alterado a qualidade das sementes determinada pelo teste de germinação. No entanto, diferindo dos resultados encontrados por Lima et al. (2013) que, avaliando o efeito da aplicação foliar de boro na qualidade de sementes de feijão comum, observaram que os tratamentos não influenciaram a porcentagem de plântulas normais, não tendo estes, alterado a qualidade das sementes determinada pelo teste de germinação.
A qualidade fisiológica das sementes de trigo oriundas dos tratamentos com aplicação de boro nos diferentes estádios de desenvolvimento da cultura, não foi prejudicada, o que evidencia que o nutriente na dosagem aplicada $\left(10 \mathrm{~kg} \mathrm{ha}^{-1}\right)$ não foi fator limitante para a qualidade das sementes produzidas.

Para o teste de frio não foi constatado efeito das diferentes épocas de aplicação de boro, bem como entre as cultivares (Tabela 4). No entanto, para o envelhecimento acelerado verificou-se que a cultivar BRS Campeiro foi superior à cultivar FUNDACEP Horizonte, não apresentando significância das diferentes épocas de aplicação de boro. Estudos realizados por Leite et al. (2011) também mostraram ausência de efeitos das aplicações de boro sobre o vigor das sementes de arroz, avaliados pelos testes de frio e de envelhecimento acelerado, permitindo inferir que a dose do elemento testada não foi prejudicial à embriogênese e, assim, não resultou na formação de sementes de diferentes níveis de vigor.

Tabela 4. Teste de frio (TF) e envelhecimento acelerado (EA) de sementes de trigo, cultivares FUNDACEP Horizonte e BRS Campeiro, provenientes da aplicação de boro em diferentes épocas.

\begin{tabular}{|c|c|c|c|c|c|c|}
\hline \multirow{3}{*}{ Época } & \multicolumn{6}{|c|}{ Cultivar } \\
\hline & Horizonte & Campeiro & & Horizonte & Campeiro & \\
\hline & \multicolumn{2}{|c|}{$\mathrm{TF}(\%)$} & Média & \multicolumn{2}{|c|}{ EA (\%) } & Média \\
\hline Sem Boro & 92 & 93 & $93 \mathrm{~A}$ & 95 & 95 & $95 \mathrm{~A}$ \\
\hline Semeadura & 96 & 92 & $94 \mathrm{~A}$ & 94 & 96 & $95 \mathrm{~A}$ \\
\hline Perfilhamento & 89 & 97 & $93 \mathrm{~A}$ & 90 & 98 & $94 \mathrm{~A}$ \\
\hline Floração & 91 & 92 & $92 \mathrm{~A}$ & 96 & 100 & $98 \mathrm{~A}$ \\
\hline $15 \mathrm{DAF}$ & 92 & 88 & $90 \mathrm{~A}$ & 96 & 94 & $95 \mathrm{~A}$ \\
\hline Média & $92 \mathrm{a}$ & $92 \mathrm{a}$ & & $94 \mathrm{~b}$ & $97 \mathrm{a}$ & \\
\hline C.V. (\%) & \multicolumn{2}{|c|}{4,13} & \multicolumn{4}{|c|}{4,14} \\
\hline
\end{tabular}

*Médias seguidas da mesma letra, minúscula na linha não diferem entre si pelo teste de $\mathrm{T}$ ( $\mathrm{p} \leq 0,05$ ), e maiúscula na coluna, não diferem entre si pelo teste Tukey $(\mathrm{p} \leq 0,05)$. 
Constatou-se interação entre os fatores de tratamento cultivar e época de semeadura para comprimento de parte aérea (Tabela 5). A cultivar BRS Campeiro apresentou resultados superiores de comprimento de parte aérea em relação à cultivar FUNDACEP Horizonte, em todas as épocas de manejo, com exceção da aplicação 15 dias após a floração (15 DAF), em que ambas as cultivares não diferiram entre si. Observou-se, no entanto, que o comprimento da parte aérea das plântulas de trigo da cultivar BRS Campeiro não sofreu efeito da aplicação de boro em nenhuma das épocas aplicadas, enquanto que a cultivar FUNDACEP Horizonte obteve incremento no comprimento da parte aérea das plântulas. Observando-se a variável comprimento de raiz (Tabela 5), a cultivar BRS Campeiro obteve resultados superiores em relação à cultivar FUNDACEP Horizonte.

Tabela 5. Comprimento de parte aérea (CPA) e de raiz (CR) de plântulas das cultivares de trigo FUNDACEP Horizonte e BRS Campeiro, submetidas a diferentes épocas de aplicação com boro.

\begin{tabular}{|c|c|c|c|c|c|c|}
\hline \multirow{2}{*}{ Época } & \multicolumn{2}{|c|}{$\mathrm{CPA}(\mathrm{cm})$} & \multirow{2}{*}{ Média } & \multicolumn{2}{|c|}{$\mathrm{CR}(\mathrm{cm})$} & \multirow{2}{*}{ Média } \\
\hline & Horizonte & Campeiro & & Horizonte & Campeiro & \\
\hline Sem Boro & 1,98 b B & 3,15 a $\mathrm{A}$ & 2,56 & 4,54 & 5,52 & $5,03 \mathrm{~A}$ \\
\hline Semeadura & $2,52 \mathrm{~b} \mathrm{~A}$ & 3,24 a A & 2,88 & 4,78 & 5,54 & $5,16 \mathrm{~A}$ \\
\hline Perfilhamento & $2,32 \mathrm{~b} \mathrm{AB}$ & 3,26 a $\mathrm{A}$ & 2,79 & 4,45 & 5,43 & $4,94 \mathrm{~A}$ \\
\hline Floração & $2,30 \mathrm{~b} \mathrm{AB}$ & 3,24 a A & 2,77 & 4,62 & 5,51 & $5,06 \mathrm{~A}$ \\
\hline $15 \mathrm{DAF}$ & 2,57 a $\mathrm{A}$ & 2,76 a $\mathrm{A}$ & 2,66 & 4,65 & 5,50 & $5,08 \mathrm{~A}$ \\
\hline Média & 2,2 & 3,24 & & $4,62 \mathrm{~b}$ & $5,51 \mathrm{a}$ & \\
\hline C.V. $(\%)$ & \multicolumn{2}{|c|}{9,10} & \multicolumn{4}{|c|}{4,36} \\
\hline
\end{tabular}

*Médias seguidas da mesma letra, minúscula na linha não diferem entre si pelo teste de $\mathrm{T}$ ( $\mathrm{p} \leq 0,05)$, e maiúscula na coluna, não diferem entre si pelo teste Tukey $(\mathrm{p} \leq 0,05)$.

Apesar da grande importância do boro sobre o crescimento de raiz no presente trabalho não foi observado efeito da aplicação de boro para essa variável. Apesar da grande importância do boro sobre o crescimento de raiz, no presente trabalho não foi observado influência da aplicação de boro para esse parâmetro. Dados que concordam com os observados por Fageria (2000), que não obteve resultados significativos em relação ao comprimento das raízes de plantas de feijão, milho, soja e trigo quanto ao fornecimento de diferentes doses de boro via solo.

\section{CONCLUSÃO}

A adubação de trigo com $10 \mathrm{~kg} \mathrm{ha}^{-1}$ de boro na forma de bórax nas diferentes épocas de aplicação, não prejudica rendimento, germinação e vigor das sementes das cultivares de trigo FUNDACEP Horizonte e BRS Campeiro. O peso volumétrico das sementes só teve redução na aplicação no perfilhamento para a cultivar FUNDACEP Horizonte e na floração para a cultivar BRS Campeiro.

\section{REFERÊNCIAS BIBLIOGRÁFICAS}

ALMEIDA, G.M.; CANTO, M.W.; NETO, A.B.; COSTA, A.C.S. Resposta da cultura de sementes de capim-mombaça a épocas e doses de adubação de boro. Semina: Ciências Agrárias, v. 36, n. 3, p. 1545-1558, 2015.

AMBROSANO, E.J.; AMBROSANO, G.M.B.; WUTKE, E.B.; BULISANI, E.A.; MARTINS, A.L.M.; SILVEIRA, L.C.P. Efeitos da adubação nitrogenada e com micronutrientes na qualidade de sementes do feijoeiro cultivar IAC-Carioca. Bragantia, v. 58, n. 2, p. 393-399, 1999.

BEVILAQUA, G.A.P, SILVA-FILHO, P.M.; POSSENTI, J.C. Aplicação foliar de cálcio e boro e componentes de rendimento e qualidade de sementes de soja. Ciência Rural, v.32, n.1, p.31-34. 2002.

BRASIL. Ministério da Agricultura, Pecuária e Abastecimento. Regras para análise de sementes. Ministério da Agricultura, Pecuária e Abastecimento. Secretaria de Defesa Agropecuária. Brasília, DF: Mapa/ACS. 395p. 2009.

CÍCERO, S.M.; VIEIRA, R. D. TESTE DE FRIO. In: Vieira RD, Carvalho NM (Ed.). Testes de vigor em sementes. Jaboticabal: FUNEP, p.151-164. 1994.

COMPANHIA NACIONAL DE ABASTECIMENTO. Acompanhamento de safra brasileira de grãos: Nono levantamento grãos safra 2014/2015 - junho 2015. Disponível em:<http://www.conab.gov.br/OlalaCMS/uploads/arquivos/1 5_06_11_09_00_38_boletim_graos_junho_2015.pdf> Acesso em: 25 de junho 2015.

DELOUCHE, J. C.; BASKIN, C. C. Accelerated aging techniques for predicting the relative storability of seed lots. Seed Science and Technology, v.1, p.427-52. 1973.

FAGERIA, N.K. Níveis adequados e tóxicos de boro na produção de arroz, feijão, milho, soja e trigo em solo de cerrado. Revista Brasileira de Engenharia Agrícola e Ambiental, v. 4, n. 1, p. 57-62, 2000.

KAPPES, C., GOLO, A.L.; CARVALHO, M.A.C. Doses e épocas de aplicação foliar de boro nas características agronômicas e na qualidade de sementes de soja. Scientia Agraria. Curitiba, v. 9, n. 3, p. 291-297, 2008.

LEITE, R.F.C.; SCHUCH, L.O.B.; AMARAL, A.S.; TAVARES, L.C. Rendimento e qualidade de sementes de arroz irrigado em função da adubação com boro. Revista Brasileira de Sementes, v. 33, n. 4, p. 785-791. 2011. 
LIMA, M.L.; CARDOSO, F.R.; GALANTE, A.H.A.; TEIXEIRA, G.C.S.; TEIXEIRA, I.R.; ALVES, S.M.F. Fontes e doses de boro na qualidade de sementes de feijão comum e mamona sob consórcio. Revista Caatinga, v. 26, n. 4, p. 3138, 2013.

LIMA, S.F.; ANDRADE, M.J.B.; CARVALHO, J.G. Resposta do feijoeiro à adubação foliar de boro, molibdênio e zinco. Ciência e Agrotecnologia, v. 23, n. 2, p. 462-467. 1999.

MALAVOLTA, E.; PIMENTEL-GOMES, F.; ALCARDE, J.C. Adubos e adubações. São Paulo: Nobel, 200p. 2002.

MALAVOLTA, E.; VITTI, G.C.; OLIVEIRA, A.S. Avaliação do estado nutricional das plantas: princípios e aplicações. 2.ed. Piracicaba: Associação Brasileira para a Pesquisa da Potassa e o Fosfato, 319p. 1997.

MARSCHNER, H. Relationship between mineral nutrition and plant disease and pests. In: Marschner, H. (Ed.) Mineral nutrition of higher plants. London. Academic Press. 369390p. 1996.

MIWA, K.; FUJIWARA, T. Boron transport in plants: coordinated regulation of transporters. Annals of Botany, v. 105, n. 7, p.1103-1108, 2010.

MORAES, L.A.C.; MORAES, V.H.F.; MOREIRA, A. Relação entre flexibilidade do caule de seringueira e a carência de boro. Pesquisa Agropecuária Brasileira, v. 37, n. 10, p. 1431-1436, 2002.

NAKAGAWA, J. Testes de vigor baseados no desempenho das plântulas. In: Krzyzanowski, F.C.; Vieira, R.D.; FrançaNeto, J.B. Vigor de sementes: conceitos e testes. Londrina: ABRATES, Cap.2, p.9-13. 1999.
OBATA, H. Micro essential elements. In: Matsuo T, Kumazawa K, Ishii R, Ishihara K, Hirata H, eds. Science of the rice plant - volume two - physiology. Food and Agriculture Police Research Center. Tokyo, 402-417p. 1995.

OLIVEIRA, J.P.M.; SCIVITTARO, W.B.; CASTILHOS, R.M.V.; OLIVEIRA FILHO, L.C.I. Adubação fosfatada para cultivares de mamoneira no Rio Grande do Sul. Ciência Rural, v. 40, n. 8, p. 1835 - 1839, 2010.

SCHEUER, P. M.; FRANCISCO, A.; MIRANDA, M. Z.; LIMBERGER, V. M. Trigo: Características e Utilização na panificação. Editorial. Revista Brasileira de Produtos Agroindustriais, v. 13, n. 2, p. 211-222, 2011.

SCIVITTARO, W.B., MACHADO, O.M. Adubação e calagem para a cultura do arroz irrigado. In: Gomes AS, Magalhães Junior AM. (Ed.). Arroz irrigado no sul do Brasil. Brasília: Embrapa Informações Tecnológicas. p. 259-297. 2004.

STRECK, E.V.; KÄMPF, N.; DALMOLIN, R.S.D.; KLAMT, E.; NASCIMENTO, P.C.; SCHNEIDER, P.; GIASSON, E.; PINTO, L.F.S. Solos do Rio Grande do Sul. 2.ed. Porto Alegre, EMATER/RS-ASCAR, 222p. 2008.

USDA - United States Department Of Agriculture. Disponível em: <http://www.fas.usda. gov/psdonline/psdQuery.aspx>. .

WRUCK, F. J.; COBUCCI, T.; STONE, L. F. Efeito do tratamento de sementes e da adubação foliar com micronutrientes na produtividade do feijoeiro. Santo Antônio de Góias: Embrapa Arroz e Feijão. 2004.

YAU, S.W.; RYAN, J. Boron toxicity tolerance in crops: a viable alternative to soil amelioration. Crop Science, v. 48, n. 3, p. 854-865, 2008. 